\title{
Desenvolvimento da agricultura familiar: investigação sobre o espaço rural e o território como referência para estudar o caso do município de Terenos, MS
}

\author{
Development of family agriculture: research on rural issues and the territory as a \\ reference to study the case of Terenos, MS
}

\section{Développement de l'agriculture familiale: la recherche sur les questions rurales et le territoire comme une référence pour étudier le cas de la municipalité de Terenos, MS}

El desarrollo de la agricultura familiar: la investigación en temas rurales y el territorio como una referencia para estudiar el caso del municipio de Terenos, MS

\author{
Thelma Lucchese Cheung* \\ (thelma.lucchese@gmail.com)
}

\author{
Recebido em 15/08/2012; revisado e aprovado em 17/10/2012; aceito em 15/01/2013
}

\begin{abstract}
Resumo: Para melhor entender como anda o processo de desenvolvimento local do município de Terenos, localizado no Estado de Mato Grosso do Sul, utilizou-se um aporte teórico territorialista. Desde a década de 1920, os primeiros agricultores de origem europeia foram assentados no local, e a sua organização deu origem à Colônia Velha e Nova. Representando uma das aglomerações mais antigas de agricultores familiares do Estado, os produtores daquelas colônias foram entrevistados estabelecendo-se como objetivo identificar suas potencialidades locais, diferenciação, interação com o território, bem como suas principais dificuldades competitivas. Embora se sustentem pelas atividades de produção agropecuárias, os agricultores declararam que os problemas relacionados à comercialização e à coordenação das cadeias nas quais têm participação são os maiores entraves na busca pelo desenvolvimento e destaque no mercado que desejam.
\end{abstract}

Palavras-chave: Agricultura familiar. Dificuldades no desenvolvimento. Diferenciação através do território.

Abstract: To understand how is the process of local development in Terenos, located in the State of Mato Grosso do Sul, we used a theoretical territorialist. Since the 1920s the first family agricultures of European origin were settled in place and your organization gave rise to the Old Colony and New Colony. Representing one of the oldest settlements of small farmers of the State, the interviews were conducted with producers of those colonies had as its objective to identify potential sites, differentiation, interaction with the territory, as well as its main competitive difficulties. Although supporting the activities of agricultural production, the family farming stated that the problems related to the marketing and coordination of the chain which has a stake are the biggest obstacles in the pursuit of development and prominence in the market who wish to

Key words: Family agriculture. Difficulties in developing. Territory differentiations.

Résumé: Pour mieux comprendre comment est le processus de développement local dans la municipalité de Terenos, situé dans l'État du Mato Grosso do Sul, nous avons utilisé un territorialiste théorique. Depuis les années 1920, les premiers agriculteurs d'origine européenne ont été réglés en place et votre organisation a donné lieu à la colonie Ancien et le Nouveau. Représentant une des plus anciennes colonies de petits agriculteurs de l'État, les entrevues ont été menées avec les producteurs de ces colonies avait pour objectif d'identifier les sites potentiels, la différenciation, l'interaction avec le territoire, ainsi que ses principales difficultés concurrentielles. Bien que soutenant les activités de production agricole, les agriculteurs ont déclaré que les problèmes liés à la commercialisation et la coordination de la chaîne qui détient une participation sont les principaux obstacles à la poursuite du développement et de l'importance dans le marché qu'ils veulent.

Mots-clés: L'agriculture familial. Les difficultés dans le développement. La différenciation à travers le territoire.

Resumen: Para entender mejor cómo es el proceso de desarrollo local en el municipio de terenos, que se encuentra en el Estado de Mato Grosso do Sul, se utilizó una territorialista teórico. Desde la década de 1920 los primeros agricultores de origen europeo se establecieron en su lugar y su organización dio origen a la Colonia Antiguo y el Nuevo. En representación de uno de los más antiguos asentamientos de pequeños agricultores del Estado, se realizaron las entrevistas con los productores de las colonias tuvo como objetivo identificar los sitios potenciales, la diferenciación, la interacción con el territorio, así como sus dificultades competitivas principales. A pesar de apoyar las actividades de producción agrícola, los agricultores declararon que los problemas relacionados con la comercialización y la coordinación de la cadena que tiene una participación son los mayores obstáculos en la búsqueda del desarrollo y protagonismo en el mercado que ellos quieren.

Palabras clave: Agricultura familiar. Dificultades en el desarrollo. La diferenciación a través del territorio.

\footnotetext{
* Universidade Federal de Mato Grosso do Sul (UFMS), Campo Grande, MS, Brasil.
} 


\section{Introdução}

No Censo Agropecuário de 2006, realizado pelo Instituto Brasileiro de Geografia e Estatística (IBGE), foram identificados 4.367.902 estabelecimentos da agricultura familiar, representando $84,4 \%$ dos estabelecimentos brasileiros. Segundo o Ministério do Desenvolvimento Agrário (MDA, 2009), a agricultura familiar é responsável por $10 \%$ do Produto Interno Bruto (PIB) do país, tendo uma produção correspondente a 54 bilhões de reais, significando $38 \%$ do valor bruto gerado pela agricultura.

Cabe destacar que, no Brasil, até a década de 1990, os termos usualmente utilizados para qualificar os agricultores familiares eram o de pequeno produtor, produtor de subsistência ou produtor de baixa renda. Em decorrência das lutas do movimento sindical por crédito, pela melhoria de preços dos seus produtos, pela busca por formas de comercialização diferenciadas e pela implementação da regulamentação constitucional da previdência social rural é que essas denominações foram dando lugar para a de agricultura familiar.

O mundo rural é caracterizado, historicamente, por se organizar em torno da produção de alimentos, cabendo aos agricultores familiares uma importante missão de abastecimento das sociedades a partir daquilo que é produzido nas unidades de produção. Segundo Zibetti (2005), existe uma teoria tridimensional sobre a função do espaço rural, a qual relaciona: a dimensão da função econômica da terra, destacando a importância do valor da alimentação e bens advindos da atividade rural; a dimensão da função social da terra, relacionada à subsistência e empregabilidade como direitos humanos de toda a coletividade; e a dimensão da função ecológica da terra, a cuja sustentabilidade estaria ligada.

De acordo com Guanziroli (2001), as discussões sobre os modelos produtivos comandados pela agricultura familiar ganham destaque, pois são impulsionadas pelos debates sobre o desenvolvimento sustentável, a geração de emprego e renda, a segurança alimentar e o desenvolvimento local.

Especificamente no Estado do Mato Grosso do Sul, o MDA (2012), com base no Censo Agropecuário 2006 do IBGE, revelou que o número de estabelecimentos da agricultura familiar é de 41.104, correspondendo a $65 \%$ das propriedades rurais. Esses estabelecimentos são responsáveis por $46 \%$ do pessoal ocupado no meio rural, com 97.431 pessoas, e $14 \%$ do Valor Bruto da Produção Agropecuária. No que concerne às atividades produção agropecuária, a agricultura familiar sul mato-grossense responde por $77 \%$ da produção de mandioca, $56 \%$ de feijão, $68 \%$ de café e $56 \%$ da produção de leite que é distribuído aos laticínios e consumido diariamente pelas famílias daquele Estado.

Considerando-se, então, a importância do papel da agricultura familiar no desenvolvimento de um país e sabendo-se que o município de Terenos no MS se sustenta economicamente através de atividades de produção agropecuária comandadas por produtores familiares organizados em duas colônias tradicionais, decidiu-se entrevistar aqueles agricultores para melhor compreender sua contribuição para o desenvolvimento do território rural do qual fazem parte.

Devido à complexidade do estudo dos territórios rurais, julgou-se interessante utilizar como referencial teórico conceitos sobre território, espaço rural e sistemas agroalimentares localizados. Por fim, cabe destacar que os resultados deste trabalho estão inseridos em outro maior, financiado pelo $\mathrm{CNPq}$, o qual teve como principal objetivo estudar os modelos de gestão de cadeia produtivas com participação da agricultura familiar.

\section{Espaço rural}

Boiser (1997) afirma que o desenvolvimento territorial consiste numa expressão ampla, que inclui o desenvolvimento do espaço rural, onde se encontram as pequenas localidades, as mudanças de ordem socioeconômica e de caráter estrutural, e os sistemas e processo decisórios, que teriam de ser democraticamente construídos, com o território, comunidade e sociedade.

No aspecto da agricultura familiar, o espaço rural está associado à ideia central de que o território possui um tecido social, uma organização complexa traçada por laços que vão muito além de seus atributos naturais. $\mathrm{O}$ espaço rural onde se reside representa uma trama de relações com raízes históricas, 

como referência para estudar o caso do município de Terenos, MS

configurações políticas e identidades que desempenham um papel ainda pouco conhecido no próprio desenvolvimento econômico (ABRAMOVAY, 2001).

O espaço rural é, então, um território que se inclina sobre o sentido de enraizamento, o sentimento de pertencimento, em relação aos laços afetivos e morais que os grupos tecem com o solo onde nasceram e estão sepultados seus antepassados (CLAVAL, 1996).

Quando se utiliza o termo dinâmica territorial rural, compreende-se este como o ambiente onde é possível utilizar, ao mesmo tempo, as potencialidades próprias do território e o aproveitamento das oportunidades externas (SCHNEIDER; MORAES, 2009). As articulações entre território, identidade, cultura e mercado formam laços de proximidade e interdependência, representando vantagens competitivas potenciais aos empreendimentos da agricultura familiar tradicional.

Levando-se em conta as relações sociais do território e as estratégias de valorização de produtos locais, como política de diferenciação, os Sistemas Agroalimentares Localizados (SYAL) constituem uma importante ferramenta estratégica para a agregação de valor aos produtos locais. $\mathrm{O}$ desenvolvimento territorial enfatiza a melhoria da qualidade e a agregação de valor aos produtos locais, a diversificação de atividades produtivas como estratégias socioeconômicas que podem ser instrumentalizadas e mediadas através de Sistemas Produtivos Localizados (SPL). A construção coletiva dos recursos territoriais nesses sistemas e aglomerados depende da interação e cooperação dos atores envolvidos nas cadeias produtivas.

\section{Sistemas Agroalimentares Localizados}

Os Sistemas Agroalimentares Localizados (SYAL) têm como base três linhas de pesquisa, a (i) primeira voltada à investigação sobre o papel de pequenas empresas ou agroindústrias na evolução da agricultura em países em desenvolvimento; a (ii) segunda linha de pesquisa nasce a partir da revisão dos distritos marshallianos, cuja ênfase está na concentração geográfica e na organização em rede de pequenas indústrias especializadas; a (iii) terceira se ocupa especialmente de questões relacionadas à qualidade dos produtos agroalimentares, certificações de origem, valorização, entre outros (MUCHNIK et al., 2007).

Ainda em relação às linhas de pesquisa citadas, a segunda linha mencionada, relacionada aos Distritos Industriais de Marshall, deu origem a duas escolas principais, uma anglo-saxã, que utilizou o conceito hoje conhecido como o de clusters, e uma escola francesa, que utilizou o conceito de Sistemas Agroalimentares Localizados.

Benko, Dunford e Lipietz (1996) defendem que a revisão da teoria marshalliana dos distritos industriais marca um rompimento com o estruturalismo global e com a teoria determinista do desenvolvimento. As teorias surgidas têm como precursoras as pesquisas de Arnaldo Bagnasco, Carlo Trigilia, Sebastiano Brusco e Giacomo Becattini sobre a Terceira Itália, e o termo cunhado por Arnaldo Bagnosco começou a ser utilizado no final da década de 1970, e está relacionado à geografia italiana. Naquela época, o reduzido progresso econômico do sul da Itália, chamado de Segunda Itália, era visível. Já o noroeste ou Primeira Itália defrontava-se com uma profunda crise, enquanto o nordeste e o centro sul da Itália destacavam-se pelo rápido crescimento (SCHMITZ, 1997).

Dessa forma, o conceito de distrito industrial foi retomado para explicar a dinâmica de crescimento das regiões do nordeste e centro sul da Itália, onde os recursos "territoriais" estariam baseados, sobretudo, no estabelecimento de redes sociais e da confiança construída pelos atores através da sua identificação com o território. Portanto, dentro do contexto de SYAL, tem-se que a noção de territorialidade é chave para a compreensão de dinâmicas econômicas e sociais. Pecqueur (1992) defende que, para a compreensão de territorialidade, faz-se necessário levar em conta quatro elementos: o sentimento de pertencimento; a transmissão dos saberes implicitamente entendidos como um patrimônio cultural da comunidade; o efeito permanente; e a importância dos atores individuais.

Vale acrescentar que, nas traduções do conceito de SYAL, defende-se a ideia de que a proximidade geográfica diminuiria os custos de transação, o que poderia favorecer a confiança e a organização em torno de um "espírito de concorrência-cooperação". A identidade territorial, nesse sistema, funcionaria como 
mediadora e facilitadora das relações entre os agentes, no caso os agricultores familiares tradicionais. Tal identidade territorial é construída pela ação coletiva dentro dos processos de formação dos Sistemas Agroalimentares Localizados, pelo compartilhamento de valores comuns, hábitos, experiências históricas e pelas relações de confiança (PECQUEUR, 1992; BOUCHER; REQUIER-DESJARDINS, 2002; REQUIER-DESJARDINS; BOUCHER; CERDAN, 2003).

Os SYAL são definidos como organizações de produção e de serviço com atividades agrícolas e empresas agroalimentares, as quais se associam, por suas características e seu funcionamento, em um território específico. O meio ambiente, os produtos, os homens, suas técnicas, seus comportamentos alimentares, suas instituições, suas redes de relacionamentos se combinam em um território produzindo uma forma de organização agroalimentar específica em uma escala espacial dada (MUCHNIK, 2006). Os Sistemas Agroalimentares Localizados seriam dinamizadores de externalidades específicas, como a integração territorial, as articulações técnicas e tecnológicas envoltas na produção de um mesmo produto, na cooperação e na troca de informações (AZEVEDO, 1996).

Segundo Ambrosini, Filippi e Miguel (2008), o estudo de SYAL remete a projetos de investigação a respeito do papel da agroindústria rural (AIR) na economia da agricultura familiar, principalmente em países da América Latina, onde os agricultores rurais, por meio da agroindústria rural, poderiam reter uma parcela maior do valor de seus produtos, através de seu processamento.

Todo esse esforço poderia representar a luta contra a pobreza e marginalização, uma vez que os agricultores familiares poderiam se tornar mais competitivos Requier-Desjardins (2006).

As relações de produção dentro dos SYAL são representadas por uma rede dupla, arranjada da seguinte forma: horizontalmente, referente ao plano territorial, envolvendo tanto atividades agrícolas e não agrícolas, com instituições locais e saberes locais. E verticalmente, com relação à cadeia alimentar, através da comercialização, condicionando o mercado e o consumo (AMBROSINI; FILIPPI; MIGUEL, 2008).
De acordo com a literatura, os laços de proximidade e de pertencimento ao lugar, as técnicas de manejo diferenciadas, o processo de cultivo baseado em redes sociais, instituições locais e a transmissão de saber-fazer específico são diferenciais competitivos da produção familiar (REQUIER-DESJARDINS; BOUCHER; CERDAN, 2003).

Foram realizadas importantes contribuições nas diversas escolas de pensamento para a análise das atuais aglomerações produtivas, mas ainda é preciso avançar na consolidação de uma abordagem teóricometodológica para o estudo e formulação de uma tipologia que tenha como intuito principal expressar as origens, formas, similaridades e complexidades das dinâmicas das aglomerações produtivas locais nos territórios rurais (REQUIER-DESJARDINS, 1999, apud SCHNEIDER; MORAES, 2009).

Pesquisas sobre Sistemas Agroalimentares Locais baseadas em formas de desenvolvimento endógeno marcam a emergência do território como unidade de análise de arranjos econômicos, segundo Wilkinson (2006), a possibilidade de vinculação de uma marca que caracterize a identificação dos produtos de um Sistema Agroalimentar Localizado a um território, por exemplo, um selo de qualidade ou origem, representa vantagens competitivas. Para os autores, esses produtos responderiam a uma demanda crescente por alimentos considerados diferenciados, valorização do território, da produção familiar, da possibilidade de fazer progredir o pequeno agricultor, do estímulo às práticas de produção mais sustentáveis.

Para Muchnick (2006), esses alimentos poderiam fazer menção aos produtos que possuem atributos positivos em relação a alimentos mais saudáveis (naturais ou orgânicos), ao meio-ambiente (produtos da agricultura ecológica ou orgânica, provenientes de projetos de desenvolvimento local sustentável), ao aspecto social (produtos oriundos da agricultura familiar ou assentamento de reforma agrária) e ao aspecto cultural (alimentos artesanais ou de gastronomia típica regional) pelo ponto de vista do consumidor, devido as suas características diferenciadas.

Com base no que foi apresentado neste breve referencial conceitual, a seguir são apresentadas informações acerca das especi- 
ficidades dos sistemas de produção de alguns produtores familiares organizados em duas colônias tradicionais de Terenos, no Estado de Mato Grosso do Sul.

\section{O caso de Terenos no estado de Mato Grosso do Sul - cadeias produtivas coman- dadas por agricultores familiares da região}

Este artigo consiste em uma pesquisa qualitativa exploratória com base em dados primários. Um questionário estruturado foi elaborado e aplicado junto a 50 pequenos produtores rurais do município de Terenos, que fazem parte da Colônia Velha e Colônia Nova. Cabe acrescentar que a primeira formação das colônias aconteceu na década de 1920 (colônia velha), com objetivo de assentar agricultores com origem europeia e garantirlhes um meio de sustento através da produção rural. A realização das entrevistas teve como intuito obter informações sobre os sistemas de produção e as influências dos antepassados, sua organização e sobre as formas de comercialização daquilo que é produzido por aqueles agricultores familiares.

Constatou-se que os agricultores familiares, através de práticas simples, conseguem resgatar atividades que preservem a sua identidade cultural. Produtos que, originalmente, eram produzidos apenas para o autoconsumo, foram transformados em mercadorias, ou seja, o saber-fazer passado entre as gerações é apresentado aos consumidores através da produção de queijos e doces artesanais. Como em grande parte das produções de pequenas propriedades rurais, a produção das colônias é diversificada, pois disso depende sua inserção no mercado. Naquela região, pôde ser observada a produção de milho, canade-açúcar, banana, além de hortas orgânicas e leite de vaca.

Para aumentar seu poder de barganha e tentar diversificar os produtos oferecidos no mercado, os pequenos agricultores justificaram a diversificação das suas atividades produtivas. A produção artesanal e a agricultura orgânica ganham importância como impulsionadoras da renda daqueles agricultores familiares uma vez que seus produtos são vendidos nas feiras da capital do Estado. Contudo ficou evidente que os produtores necessitam de auxílio, externo à sua organização coletiva, o qual lhes permita ter uma maior eficiência produtiva, bem como chegar até o consumidor final através de canais de distribuição diferentes daqueles que já adotam.

Através das entrevistas, pôde-se perceber que seus maiores entraves são a falta a competitividade e os problemas relacionados à comercialização (sobretudo no que concerne ao pouco conhecimento sobre as demandas dos consumidores). Percebeu-se que sua identidade territorial ainda não está representada nas suas produções. Dessa forma, como meio de promover ainda mais o desenvolvimento daquele território, sugere-se como ações a tentativa de atribuição de selos de origem e qualidade aos produtos das colônias e a realização de estudos que permitam uma melhor compreensão sobre o perfil e as demandas de consumidores que valorizam aquele tipo de produção para, por exemplo, saber quais são os pontos de venda mais desejados por eles para encontrar aqueles produtos e quais os melhores meios de com eles estabelecer comunicação.

Sabe-se que a valorização do conhecimento e do saber acumulado e passado pelos agricultores, nos processos e técnicas de gestão do meio natural, podem representar vantagens competitivas aos agricultores familiares. A realização de feiras e a atribuição de selos de qualidade poderiam promover a valorização local, manter e melhorar aquela organização coletiva, o que se faz necessário.

Os agricultores familiares, fazendo uso da sua relação com o território e buscando o desenvolvimento de toda uma região, reforçam a importância dos aspectos territoriais e históricos locais.

As declarações dos agricultores familiares e o conhecimento das suas atividades de produção (potencialidades e principais gargalos) levaram-nos a concluir que, para um maior desenvolvimento local, será necessário investir em processamento dos produtos produzidos, fazendo a interface entre a produção e o consumo, através das articulações das agroindústrias rurais (AIR). Tudo isso para agregar valor e diferenciar sua atividade de produção agropecuária. As Agroindústrias rurais, segundo Muchinick (2006), são capazes de: (i) aumentar significativamente o valor acrescentado obtido pelos agricultores familiares; (ii) melhorar a renda deles; (iii) 
gerar um número significativo de postos de trabalho nas zonas rurais; e (iv) contribuir para a segurança alimentar da população, tanto em áreas rurais como em áreas urbanas.

De qualquer forma, para melhorar ainda mais o potencial produtivo daquele território e promover seu melhor desenvolvimento, faz-se ainda necessário que os agricultores busquem, por formas mais eficazes de coordenação e cooperação, um beneficiamento conjunto.

\section{Considerações finais}

Segundo o Ministério do Desenvolvimento Agrário (MDA), há uma estimativa para que, ainda em 2010, a produção advinda dos agricultores familiares no Brasil, chegue à ordem de 18 milhões de toneladas de alimentos/ano. Dados como este mostram não só a representatividade que a Agricultura Familiar tem para a economia do país, como também a preocupação, cada vez maior, de órgãos e instituições quanto ao papel do agricultor familiar, com o que se caminha para a transformação do campo brasileiro em um espaço de cidadania, produção e sustentabilidade, para que determinados agricultores, alcançando um desenvolvimento sustentável, consolidem sua permanência nos empreendimentos rurais.

Através de um levantamento teórico, concluiu-se que a sustentabilidade das produções comandadas por agricultores familiares agindo em comunidade poderia ser alcançada com a cooperação, a fim de diminuir os custos de transação; com a atribuição de valores culturais e históricos aos seus produtos, em que valores comuns, hábitos e experiências históricas são levados em consideração; com o valor imensurável ao território onde residem, atribuindo-lhe uma identidade territorial e possuindo uma relação de confiança, como redes de relacionamento.

De modo geral, os agricultores familiares necessitam de auxílio para que compreendam que suas peculiaridades são um diferencial competitivo. Desse modo, se faz necessária a união de diferentes instituições a fim de mostrar caminhos a esse tipo de agricultor. É possível observar que o relacionamento entre agricultores e instituições ligadas ao Estado está evoluindo; porém cabe a essas instituições mais do que fornecer insumos, é preciso trabalhar em conjunto, estar presente em todos os elos produtivos, inclusive na comercialização, elo mais fraco da cadeia produtiva.

Nesse contexto, a agricultura familiar necessita de novas abordagens baseadas em construções teórico-metodológicas que expliquem as dinâmicas socioeconômicas e culturais de desenvolvimento dos territórios rurais, levando em consideração as formas estruturais e institucionais de regulação e governança existentes e, ao mesmo tempo, possibilitando-lhes a modificação pela ação dos atores sociais locais.

Para permitir aos agricultores familiares de Terenos uma maior otimização das suas produções, uma das possibilidades seria a partir da utilização de seus atributos locais específicos. Tal ação poderia dar margem para a implementação de uma série de medidas que poderiam levar a um desenvolvimento sustentável e maior geração de renda, sempre preservando a cultura, a tradição e a tipicidade dos produtos.

\section{Referências}

ABRAMOVAY, Ricardo. Ruralidade e desenvolvimento regional. Gazeta Mercantil, 15 abr. 2001.

. Paradigmas do capitalismo agrário em questão. 3. ed. São Paulo: Edusp, 2007.

AMBROSINI, Larissa Bueno; FILIPPI, Eduaro Ernesto; MIGUEL, Lovois de Andrade. SIAL: análise da produção agroalimentar a partir de um aporte territorialista e multidisciplinar. Revista Ideas - Interfaces em Desenvolvimento, Agricultura e Sociedade, Rio de Janeiro, v. 2, n. 1, p. 6-31, jan./jun. 2008.

AMBROSINI, Larissa Bueno; FILIPPI, Eduaro Ernesto. Da era do desenvolvimento ao desenvolvimento rural: sistemas produtivos localizados sob a perspectiva de Karl Polany. REDES, Santa Cruz do Sul, v. 13, n. 3, p. 121-139, set./dez. 2008.

AZEVEDO, B. Développement Local: Industrie, Famille et Territoire. In: ABDELMAKI, L.; COURLET, C. (Orgs.). Les Nouvelles Logiques du Développement. Paris: L'Harmattan, 1996. p.189-203.

BENKO, Georges; LIPIETZ, Alain. (Orgs.). Les Régions qui Gagnent. Paris: Presses Universitaires de France, 1992. p. 35-55.

BOISIER, Sergio. El vuelva de una cometa: una metamorfose para una teoría del desarrollo territorial. Santiago: ILPES/CEPAL, 1997.

CLAVAL, Paul. O território na transação da pósmodernidade. Revista Géographies et Cultures, Paris, n. 20, inverno 1996.

GUANZIROLLI, Carlos et al. Agricultura familiar e re- 

como referência para estudar o caso do município de Terenos, MS

forma agrária no século XXI. Rio de Janeiro: Garamond, 2001.

INSTITUTO BRASILEIRO DE GEOGRAFIA E ESTATÍSTICA - IBGE. Disponível em: <www.ibge.gov.br>. Acesso em: 16 abr. 2010.

MINISTÉRIO DO DESENVOLVIMENTO AGRÁRIO. Mais alimentos - um plano de agricultura familiar para o Brasil. MDA, 2008/2009.

MORAES, Jorge L. Amaral de: SCHNEIDER, Sérgio. A abordagem dos Sistemas Produtivos Localizados (SPLs) Rurais e as dinâmicas sócioeconômicas de desenvolvimento dos territórios rurais da região Vale do Rio Pardo. REDES, Santa Cruz do Sul, v. 14, n. 2, p. 172-197, maio/ago. 2009.

MUCHNIK, José. Identidad Territorial y Calidad de Los Alimentos: Procesos de Calificación y Competencias de los Consumidores. In: Revista Agroalimentaria, 2006.

Sistemas Agroalimentarios Localizados: Evolución del Concepto y Diversidad de Situaciones. In: CONGRESO INTERNACIONAL DE LA RED SIAL “ALIMENTACIÓN Y TERRITORIOS", 3, Baeza (Jaén). Comunicaciones... Baeza: GYS SYAL, 2006a.

MUCHNIK, José; BIENABÉ, Estelle; CERDAN, Claire. Food Identity / Food Quality: Insights from the 'Coalho' Cheese in the Northeast of Brazil. Antropology of Food, v. 4, maio 2005.

PECQUEUR, Bernard. Territoire, Territorialité e Dé- veloppement. In: COLLOQUE INDUSTRIE ET TERRITOIRE: LES SYSTEMES PRODUCTIFS LOCALISES, Grenoble. Actes... Grenoble: IREP-D, 1992.

REQUIER-DESJARDINS, Denis. Alternativas de Desarrollo Local para el Combate de la Pobreza y de la Exclusión Social. In: CONGRESO INTERNACIONAL DE LA RED SIAL - "ALIMENTACIÓN Y TERRITORIOS", 3 , Baeza (Jaén). Comunicaciones... Baeza: GYSSYAL, 2006a.

REQUIER-DESJARDINS, Denis; BOUCHER, François; CERDAN, Claire. Globalization, Competitive Advantages and the Evolution of Production Systems: Rural Food Processing and Localized Agri-food Systems in Latin American Countries. Entrepreneurship and Regional Development, v. 15, n. 1, p. 49-67, 2003.

SCHMITZ, Hubert. Eficiência coletiva: caminho de crescimento para a indústria de pequeno porte. Ensaios FEE, Porto Alegre, v. 18, n. 2, p. 164-200, 1997.

SCHNEIDER, Sérgio; NIEDERLE, Paulo André. Agricultura Familiar e Teoria Social: a diversidade das formas familiares de produção na agricultura. In: SIMPÓSIO NACIONAL CERRADO, 9.

WILKINSON, John. A agricultura familiar face ao novo padrão de competitividade do Sistema Agroalimentar na América Latina. In: SEMINÁRIO DE CULTURA E ALIMENTAÇÃO. Anais... Vila Maria/São Paulo: SESC, 2006.

ZIBETTI, D. W. Teoria tridimencional da função da terra no espaço rural. Curitiba: Jeruá, 2005. 154p. 